\title{
HR Carinae: New spectroscopic data and physical parameters ${ }^{\star, \star \star}$
}

\author{
M. A. D. Machado, F. X. de Araújo, C. B. Pereira, and M. B. Fernandes \\ Observatório Nacional-MCT, Rua José Cristino, 77, CEP 20921-400, São Cristóvão, Rio de Janeiro-RJ, Brazil
}

Received 13 November 2001 / Accepted 29 January 2002

\begin{abstract}
We present new high-resolution observations of the LBV (or S Dor variable) HR Carinae, taken with the FEROS spectrograph in the wavelength range $\lambda$ 3900-8200. The H I and Fe II lines show P Cygni profiles while the He I lines are all in absorption. Emission lines due to [N II], [OI], Na II, Mg II, Ca II and [Fe II] were also identified. Complex structures in the absorption profiles of $\mathrm{H} \alpha, \mathrm{H} \beta$ and in most of the iron lines suggest the presence of two distinct absorption regions with expansion velocities of $30 \mathrm{~km} \mathrm{~s}^{-1}$ and $100 \mathrm{~km} \mathrm{~s}^{-1}$. From a spectral analysis of the Balmer lines with a non-LTE expanding atmosphere code, we derive for HR Carinae the following parameters: $T_{\star}=10000 \mathrm{~K}, R_{\star}=350 R_{\odot}, \dot{M}=6.8 \times 10^{-5} M_{\odot} \mathrm{yr}^{-1}$ and $\mathrm{He} / \mathrm{H}=0.4$. The comparison of the temperature obtained in this work with the values estimated by Nota et al. (1997) indicates that HR Carinae is moving redward across the H-R diagram on a short timescale, due to a new S Dor-phase.
\end{abstract}

Key words. stars: emission-line, Be - stars: variables: general - stars: individual: HR Car

\section{Introduction}

HR Car (HD90177) belongs to a small group of very massive, luminous and evolved supergiants called luminous blue variables (LBVs) or S Doradus (S Dor) variables. Such stars are characterized by irregular spectroscopic, photometric and polarimetric variations. In the H-R diagram they are located close to the Humphreys-Davidson limit (Humphreys \& Davidson 1994) and play a fundamental role in studys of the physical instabilities in this part of the diagram. They appear to move at roughly constant bolometric luminosity between a hot, minimum visual phase $\left(T_{\text {eff }} \approx 20000 \mathrm{~K}-30000 \mathrm{~K}\right)$ and a cooler, maximum visual phase $\left(T_{\text {eff }} \approx 8000 \mathrm{~K}-10000 \mathrm{~K}\right)$. In this cooler phase, also called the active or SD-phase, the LBV resembles a very luminous A-type supergiant.

LBVs are believed to represent a very short transition phase that occurs in the evolution of massive stars with initial mass $M_{\mathrm{i}} \geq 40 M_{\odot}$. The observed high massloss rates of LBVs peel off the outer H-rich atmosphere and reveal the products of CNO-cycle burning. However, the various evolutionary models do not agree about when

\footnotetext{
Send offprint requests to: M. A. D. Machado, e-mail: dora@dage0.on.br

* Based on observations made with the $1.52 \mathrm{~m}$ telescope at the European Southern Observatory (La Silla, Chile) under the agreement with the Observatório Nacional, Brazil.

$\star \star$ Tables 1 and 2 are only available in electronic form at http://www. edpsciences.org
}

exactly the LBV phenomenon should occur (Schaller et al. 1992; Langer et al. 1994; Stothers \& Chin 1996).

The spectral type of HR Car is variable. Carlson \& Henize (1979) classified this object as a B2 I PCygni that undergone irregular slow variations of approximately $1.5 \mathrm{mag}$ over a period of a few months. Ultraviolet observations made by Shore et al. (1990) suggested a B4B5 type. Walborn \& Fitzpatrick (2000), using absorptionline criteria, suggested that HR Car is a B3 Ia star, but the $\mathrm{Mg}$ II $\lambda 4481$ / He I $\lambda 4471$ ratio indicates a B6B7 spectral subtype. It should be kept in mind that HR Car, like other S Dor variables, is subject to a cyclic pattern of S Dor-phases with a time scale of years to decades (van Genderen et al. 1997). Thus, brightness, spectrum, temperature, colour, etc., are cyclically variable. With regard to the distance, in the past it has been assumed that HR Car belongs to the Carinae complex and its distance was taken as $2.5 \mathrm{kpc}$. This determination was revised by van Genderen et al. (1991), who derived a value of $5 \pm 1$ $\mathrm{kpc}$ with the aid of the reddening-distance method.

The majority of LBVs is associated with a circunstelar nebula apparently formed of matter ejected by the star and probably enriched by the products of nuclear burning. Several analyses have been reported concerning the geometry, dynamics and chemical composition of the surrounding nebula of HR Car: Hutsemékers \& van Drom (1991), Nota et al. (1995), Clampin et al. (1995). More recently Nota et al. (1997) confirmed that the nebulae is 
bipolar, relatively young, is moving fast and is composed of CNO elements.

Spectroscopic data of HR Car have also provided information about the star and the nebula. Shore et al. (1990) presented a multiwavelength analysis of ultraviolet, optical and radio observations from the Carlson \& Henize (1979) sample. They investigated in detail the spectra of HR Car and, combining UV data with the available $U B V$ photometry, they estimated a reddening of $E(B-V)=1.15 \pm 0.005$. Hutsemékers \& van Drom (1991) obtained medium-resolution and high-resolution optical spectroscopic data from HR Car that indicated that the star undergoes spectral variations similar to other LBVs and has a multiple shell, expanding atmosphere. Nota et al. (1997) carried out two sets of optical observations, from which they estimated the effective temperature of the star and observed changes in the shape of $\mathrm{H} \alpha$ profile. They have associated the changes with multiple forming shells. Infrared spectroscopic data were obtained by McGregor et al. (1988), who found cool dust around HR Car and reported a variable spectrum marked by strong [Fe II] emission lines. In addition, Morris et al. (1996) investigated permitted and forbidden iron lines in the IR spectra and found a spectral similarity between HR Car and WRA 751 (= Hen 3-591) in the $K$ band.

The aims of the present paper are: (i) to describe new, high-dispersion spectroscopic data of HR Car; (ii) to estimate relevant stellar and wind parameters of this object due to the fits of Balmer line profiles and (iii) to compare our results with previous ones, interpreting the variations on the scope of LBVs/S Dor variables. For the sake of completeness a low-resolution spectrum is also shown.

\section{Observations and reduction}

Spectroscopic high-resolution observations were performed with a Fiber-fed Extended Range Optical Spectrograph (FEROS, see Kaufer et al. 1999), with the ESO $1.52 \mathrm{~m}$ telescope in La Silla (Chile) at April 19, 1999. FEROS is a bench-mounted Echelle spectrograph with the fibers located at the Cassegrain focus with a spectral resolving power of $R=48000$ corresponding to 2.2 pixels of $15 \mu \mathrm{m}$ and with a wavelength coverage from $\lambda 3600$ to $\lambda$ 9200. A completely automatic on-line reduction is available and it was adopted by us. The exposure time was $1200 \mathrm{~s}$, leading to a $S / N$ ratio in the continuum of approximately 150 in the $\lambda 5500$ region.

The low-resolution spectrum of HR Car was obtained using a Boller \& Chivens spectrograph at the Cassegrain focus at March 12, 1998. The spectrum of HR Car was obtained with grating \#23 $\left(600 \mathrm{l} \mathrm{mm}^{-1}\right)$, providing a resolution of $4.6 \AA$ in the range $\lambda 3600-7400$. The Cassegrain spectrum was reduced using standard IRAF tasks, such as bias subtraction, flat-field normalization, and wavelength calibration. For an exposure time of 10 s the $S / N$ ratio in the continuum is aproximately 70 in the $\lambda 5500$ region.

\section{Description of the spectrum}

The FEROS spectrum of HR Car is shown in Figs. $1-5$, in the range from $\lambda 3950$ to $\lambda 8900$. The line identification was carried out using the line lists provided by Moore (1945), Garcia-Lario et al. (1998), Landaberry et al. (2001) and Fernandes et al. (2001). We also looked up two sites on the web: NIST Atomic Spectra Database Lines Form (URL physics.nist.gov/cgi/AtData/lines) and The Atomic Line List v2.04 (URL www.pa.uky.edu/peter/atomic/). Tables 1 and 2 summarize the features identified in our spectrum of HR Car. In Table 1 we present the equivalent widths $\left(W_{\lambda}\right)$ of emission lines; in the case of $\mathrm{P}$ Cygni profiles only the equivalent width of the emission component was measured. If the equivalent width is very small $\left(W_{\lambda} \leq 0.01\right)$ but the transition is surely present, only the identification is given. Table 2 shows the absorption features that have been found. Figure 6 presents the lowresolution spectra of HR Car.

Selected regions in the optical spectrum of HR Car have been discussed by Hutsemékers \& Van Drom (1991), Lopes et al. (1992), Nota et al. (1997) and Walborn \& Fitzpatrick (2000). It is marked by several P Cygni profiles from Balmer and single ionized iron lines. Almost all profiles show a complex structure superimposed on the absorption component that will be discussed in more detail later in this paper.

The He I lines presented in our spectrum are all in absorption. They are listed in Table 2. No evidence is found for the presence of He II in the spectrum of HR Car.

The [N II] transitions at $\lambda 5754.8[3 \mathrm{~F}], \lambda \lambda 6548.1$ and $6583.6[1 \mathrm{~F}]$ as well as the $[\mathrm{O} \mathrm{I}]$ multiplet at $\lambda \lambda 6300.2$ and 6363 are present, but quite weak. Nota et al. (1997) list the characteristic photospheric lines Si II $\lambda \lambda 6347,6371$ $\lambda \lambda 6379,6482$, Ne I $\lambda 6506$ and Fe I $\lambda 6614$ observed at two different epochs and compare the respective equivalent widths. All these lines are present in our spectrum, however their equivalent widths are smaller, with the exception of $\mathrm{Si}$ II $\lambda \lambda 6347,6371$ that is stronger. This doublet was used as a temperature indicator by Nota et al. (1997). We will address this point in Sect. 6. The resonance doublet of $\mathrm{Na}$ I at $\lambda \lambda 5890-5896$ is present. We also observe the $\mathrm{Mg}$ II lines $\lambda \lambda 7877,7896(\mathrm{~m} 8)$ and $\lambda \lambda 8213,8235(\mathrm{~m} 7)$ and the CaII triplet $\lambda \lambda 8498,8662$ in emission.

\subsection{The hydrogen lines}

All Balmer lines exhibit P Cygni profiles, revealing a significant variation when compared to the data obtained in 1989 by Walborn \& Fitzpatrick (2000), in which the emission component of $\mathrm{H} \epsilon, \mathrm{H} \delta$, and $\mathrm{H} \gamma$ were very weak, or almost absent. Furthermore, in our spectrum, the absorption components of $\mathrm{H} \alpha$ and $\mathrm{H} \beta$ are split into two components. This split is sharper in $\mathrm{H} \alpha$ than in $\mathrm{H} \beta$ and is not present in $\mathrm{H} \gamma$ and $\mathrm{H} \delta$, as is shown in Fig. 9. This behavior has been observed by Hutsemékers et al. (1991) 
in the Balmer and iron lines and by Nota et al. (1997) in the $\mathrm{H} \alpha$ profile obtained in their second set of observations. Both papers associate this split with the presence of two differents shells in the atmosphere of HR Car. The $\mathrm{H} \alpha$ equivalent width measured by us is very close to the constant value measured by Nota et al. (1997) in their two sets of observations.

The broad wings on the emission components of Balmer lines have been described previously by Hutsemékers et al. (1991). One possible explanation for these broad wings, observed also in P Cygni and AG Car, is the non-coherent scattering of the emission line photons by free electrons (Berbat \& Lambert 1978; Wolf \& Stahl 1982). Hutsemékers et al. (1991) rejected this argument because it presupposes different regions of formation of these lines, which according to them would not be supported by net differences in the radial velocities. They explained the broad wings as a consequence of high velocity motions in the atmosphere of HR Car. In Sect. 5, we will give some arguments in favour of formation of Balmer lines in differents regions of the extended atmosphere, reinforcing that the broad wings could be produced by eletronic scattering.

\subsection{The iron lines}

The most numerous lines present in HR Car are of single ionized iron, both permitted and forbidden. The strongest features can be identified as being due to multiplet 42 at $\lambda \lambda 4924,5018$ and 5169. There are several blends of Fe II and [Fe II] lines. Almost all Fe II features show a P Cygni profile with an absorption splitting more complex than that observed in $\mathrm{H} \alpha$. Figure 7 shows the profiles of the three lines of multiplet 42 and of some other multiplets. We can clearly see two main absorption components, for which the measured radial velocities are $30 \mathrm{kms}^{-1}$ and $100 \mathrm{kms}^{-1}$ (inner and outer shells respectively). These two absorption components were reported previously by Hutsemékers et al. (1991) with the same radial velocity and are in agreement with the structures detected by Nota et al. (1997) from the images of the nebulae. Thanks to the high resolution of FEROS, we could observe other weaker absorption components that they were not noted by Hutsemékers et al. (1991). Presumably they are associated with sub-shells.

On the other hand, we note that [Fe II] lines are in pure emission, as we can see in Fig. 8. The profiles of these lines are similar to the [Fe II] profiles of HD316285 described by Hillier et al. (1998).

\section{Summary of the model}

The spectral analysis of stars with atmospheres in expansion such as HR Car requires a specific model that solves the radiative transfer and the statistic equilibrium equations simultaneously in a non-LTE regime. Machado (1998) developed a numerical code adequate to investigate the line formation in expanding stellar atmospheres of massive stars. This code was used to study the peculiar B-type supergiant HD327083 (Machado et al. 2001). The basic assumptions are: spherical symmetry, stationary and homogeneity. The density structure $\rho(r)$ is related to the mass loss rate $\dot{M}$ and the velocity field $V(r)$ via the equation of continuity

$\rho(r)=\frac{\dot{M}}{4 \pi r^{2} V(r)}$

$\dot{M}$ is a input parameter and the velocity field is prespecified in an ad-hoc way as a $\beta$-type law

$V(r)=V_{\infty}\left(1-R_{\star} / r\right)^{\beta}$

where $V_{\infty}$ is the terminal velocity of the wind and $\beta$ is a measure of the rate of acceleration (de Koter at al. 1993).

The statistical equilibrium equations are solved using the escape probability method for calculating the source function while the transfer equation is solved using the "SEI" - Sobolev Exact Integration - method (Lamers et al. 1987). This is justified by the flow velocities observed in the wind of HR Car.

In this work it is considered that the wind begins in the sonic point localized at radius $R_{\star}$. The stellar temperature $T_{\star}$ is defined by the luminosity $L_{\star}$ and the radius $R_{\star}$ via the Stefan-Boltzmann law.

Regarding the temperature structure we have used the law suggested by Drew (1985):

$T(r)=T_{\star}\left[0.78-\left(0.51 \frac{V(r)}{V_{\infty}}\right)\right]$

We are aware that this temperature profile is more appropriate to main sequence $\mathrm{O}$ stars. Thus, we have checked the influence of the temperature law. Some simple tests with isothermal and radially decreasing ad-hoc profiles have been performed. It was found that the final result is not sensitive to similar temperature laws.

The model atmosphere in the present work consists of hydrogen and helium. For hydrogen and singly ionized helium we consider the lowest ten bound levels. For neutral helium we adopt the atomic model suggested by Almog $\&$ Netzer (1989) that includes the individual levels until $n \leq 4$ and combined levels with $5 \leq n \leq 10$.

The determination of the population numbers of the energy levels is made in a non-LTE way solving the equations of statistical equilibrium for each level considered in the atomic model. The radiative transition probabilities and the photoionization cross section for all levels considered are quoted from the Opacity Project.

The ionization collision cross sections have been obtained from the approximate formulae given by Jefferies (1968). Excitation collisional rates have been calculated from the collisional strength values. For hydrogen and singly ionized helium these values are computed using polynomials given by Giovanardi et al. (1987). For neutral helium the collision strengths are taken from Berrington \& Kingston (1987) to levels with $n \leq 4$ and from 

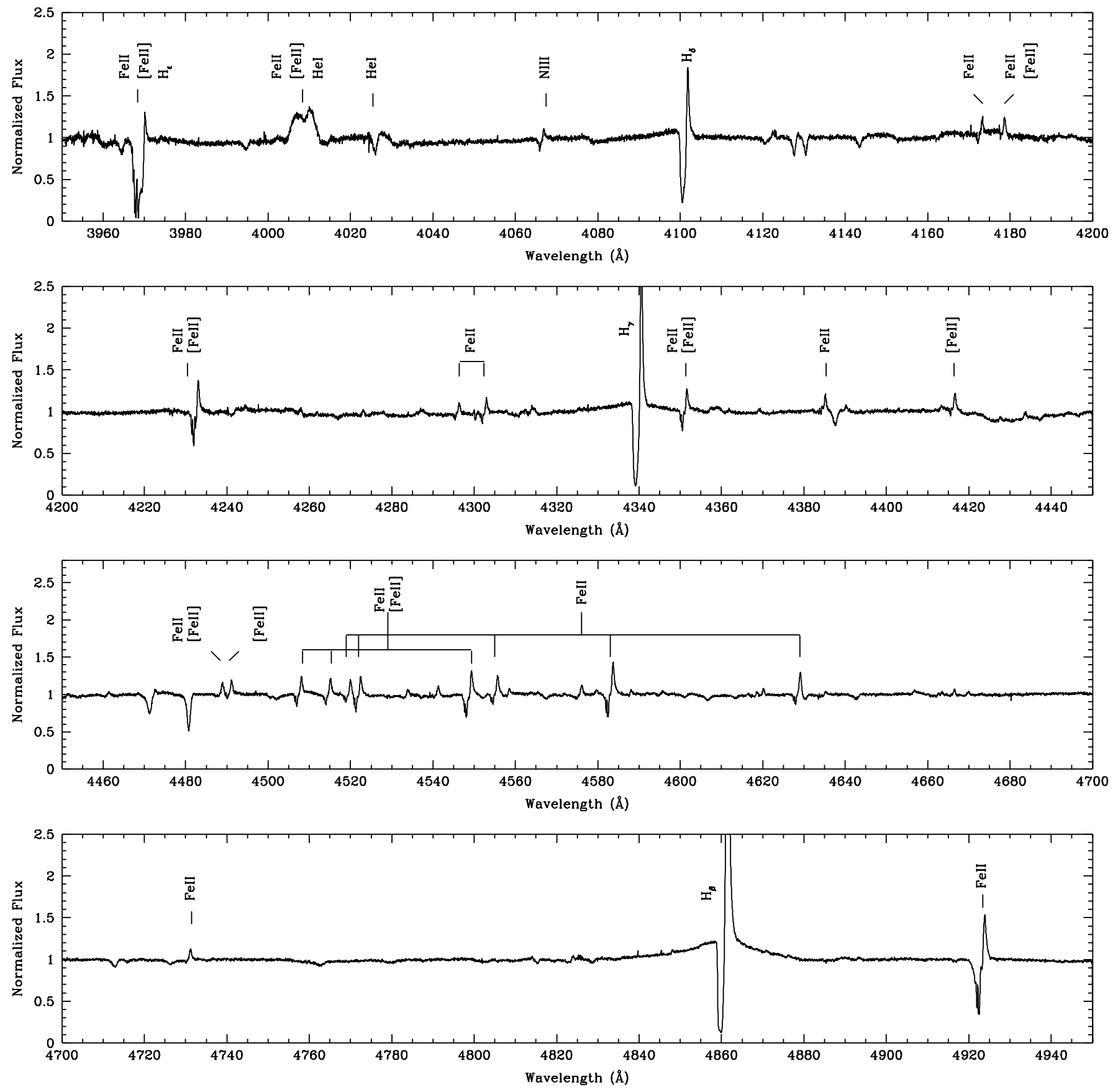

Fig. 1. High resolution spectrum of HR Car obtained with the FEROS spectrograph.

Auer \& Mihalas (1968) and Mihalas \& Stone (1968) to the other ones.

The radiation and the statistical equations systems are solved simultaneously through the wind that is divided in shells. A convergence criterium is applied in order to move from one shell to the next.

\section{Balmer line analysis}

\subsection{Methodology}

The input parameters of the model are the stellar temperature $T_{\star}$, the luminosity $L_{\star}$, the mass loss rate $\dot{M}$, the terminal velocity $V_{\infty}$, the helium abundance $A_{\mathrm{He}}$ and the free parameter of the velocity profile $\beta$. In the present work we have fixed the value of the terminal velocity, $V_{\infty}=400 \mathrm{~km} \mathrm{~s}^{-1}$, that is an average of the $V_{\text {edges }}$ of Balmer lines profiles. In order to reduce the number of parameters, we have also fixed the luminosity $\left(L_{\star}\right)$ using the value of $L_{\star}$ estimated by Hutsemékers (1994).

The strategy to obtain the line fits of HR Car was similar to that used in the analysis of HD 327083 (Machado et al. 2001). Once the luminosity was fixed, we explored a broad range in temperatures, which roughly corresponds to the domain of LBVs and Yellow Supergiants in the H$\mathrm{R}$ diagram: $31000 \mathrm{~K}-8000 \mathrm{~K}$. This range is based on the 

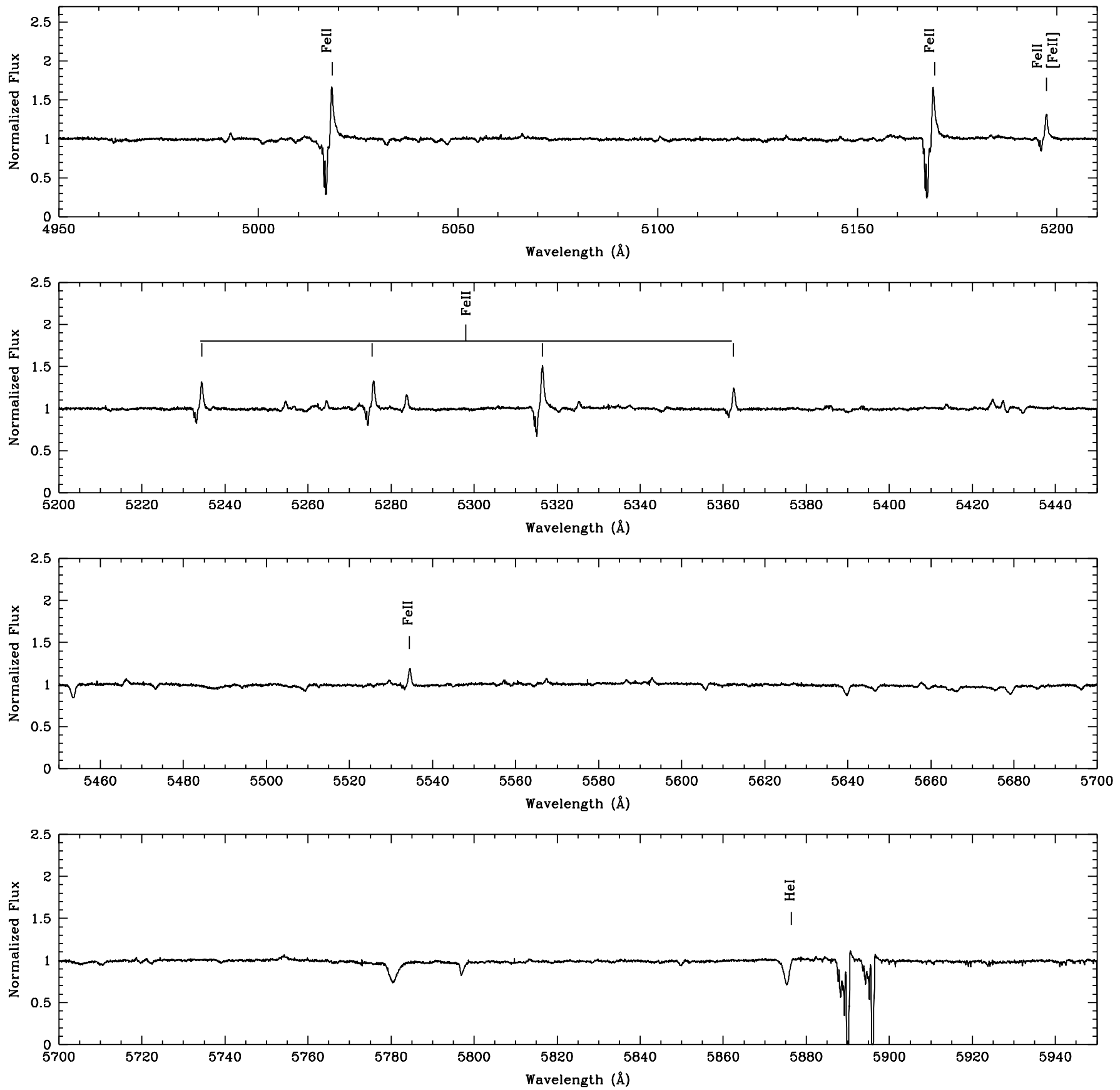

Fig. 2. High resolution spectrum of HR Car obtained with the FEROS spectrograph (continuation).

works of Gummersbach et al. (1995), Szeifert et al. (1996) and de Jager \& Nieuwenhuijzen (1997).

After that, we computed models according to the evolutionary tracks calculated by Schaller et al. (1992) from $\mathrm{M}_{\text {ZAMS }}=20 M_{\odot}$ to $120 M_{\odot}$. Each track consists of a number of points that provide stellar temperature, luminosity, mass loss rate and surface abundance ratio. These are the entry parameters for our code. The models that lead to reasonably good fits (according to a $\chi^{2}$ criterium) are taken as starting points. Then we varied the input param- eters in order to obtain better adjustments. In this work we have modelled the Balmer lines $\mathrm{H} \delta, \mathrm{H} \gamma, \mathrm{H} \beta$ and $\mathrm{H} \alpha$.

\subsection{Results}

The line fits obtained are shown in Fig. 9. The best model that produces such fits corresponds to the following set of parameters: $\dot{M}=6.5 \times 10^{-5} M_{\odot} \mathrm{yr}^{-1}, T_{\star}=$ $10000 \mathrm{~K}, L_{\star}=5 \times 10^{5} L_{\odot}, R_{\star}=350 R_{\odot}$ and $\mathrm{A}_{\mathrm{He}}=0.4$. The theoretical line profiles are directly influenced by the 

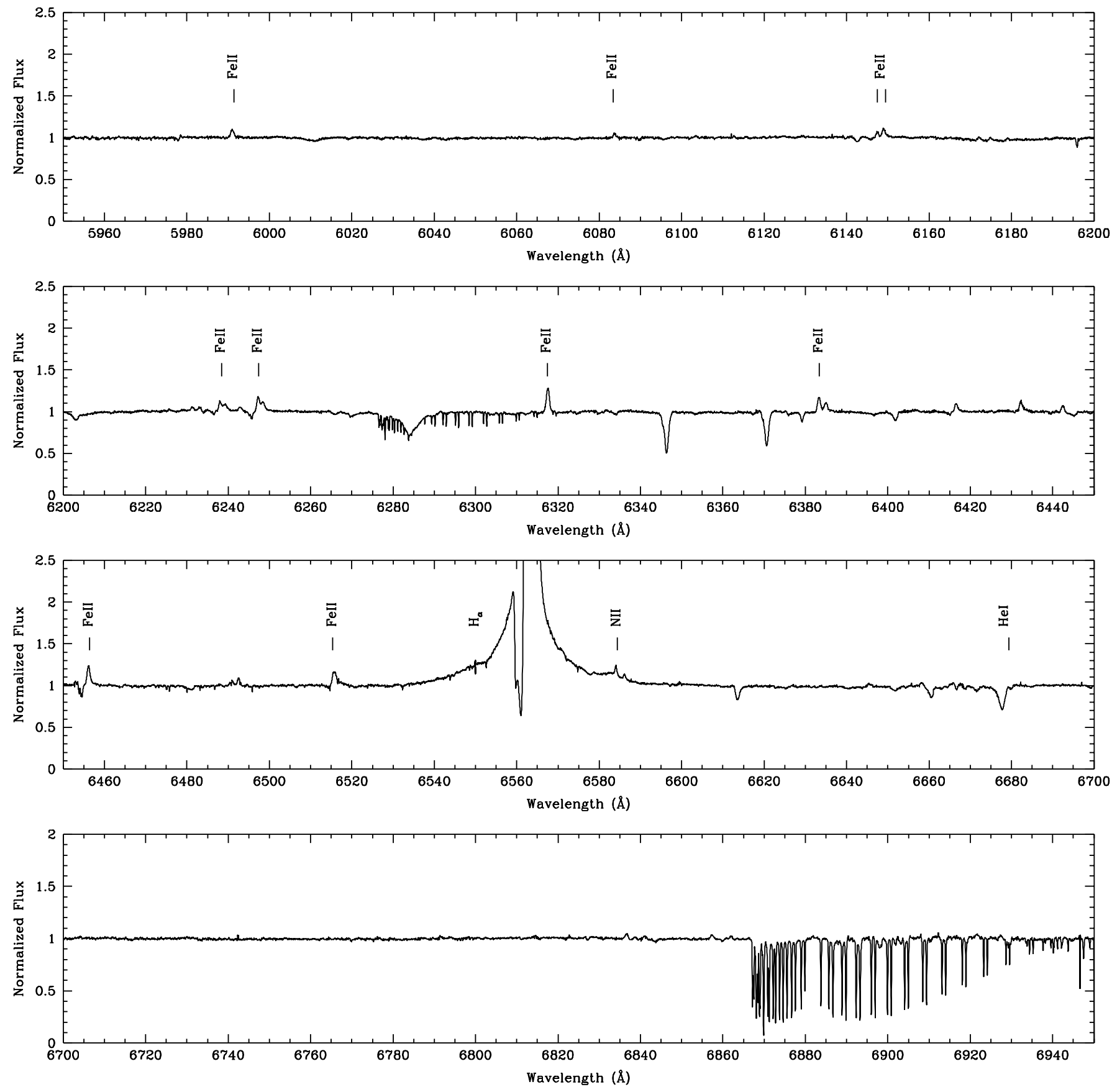

Fig. 3. High resolution spectrum of HR Car obtained with the FEROS spectrograph (continuation).

parameters $T_{\star}$ and $A_{\mathrm{He}}$. The He I lines are strongly dependent on temperature. As in HR Car these lines are all in absorption, the temperature was set in order not to produce helium lines in emission. On the other hand, the hydrogen lines are more dependent on density. Even by varying the mass loss rate, we need to decrease the quantity of hydrogen to reproduce the observed profile. This was done by increasing the helium abundance.

From Fig. 9 we can see that the emission component of the P-Cygni profiles are quite well reproduced. Concerning the blue-shifted absorption, the fits are not so good. In fact they are rather poor for $\mathrm{H} \alpha$ and $\mathrm{H} \beta$ but become better for the higher terms of the Balmer series. This behavior may be easily understood. Let us consider a transition betwen a bound lower level $i$ and a bound upper level $j$. For a certain total density, the absorption process related to the transition $i \rightarrow j$ becomes more and more difficult as $j$ increases. Thus the higher terms of the Balmer series should be formed in higher density regions, i.e. in regions close to the star. We also expect that in the inner regions the wind is more spherically symmetrical than in the outer regions. In addition, we note that our model assumes a spherical expansion. Consequently, it is not surprising that the $\mathrm{H} \gamma$ and $\mathrm{H} \delta$ profiles (that are produced in a more or less 

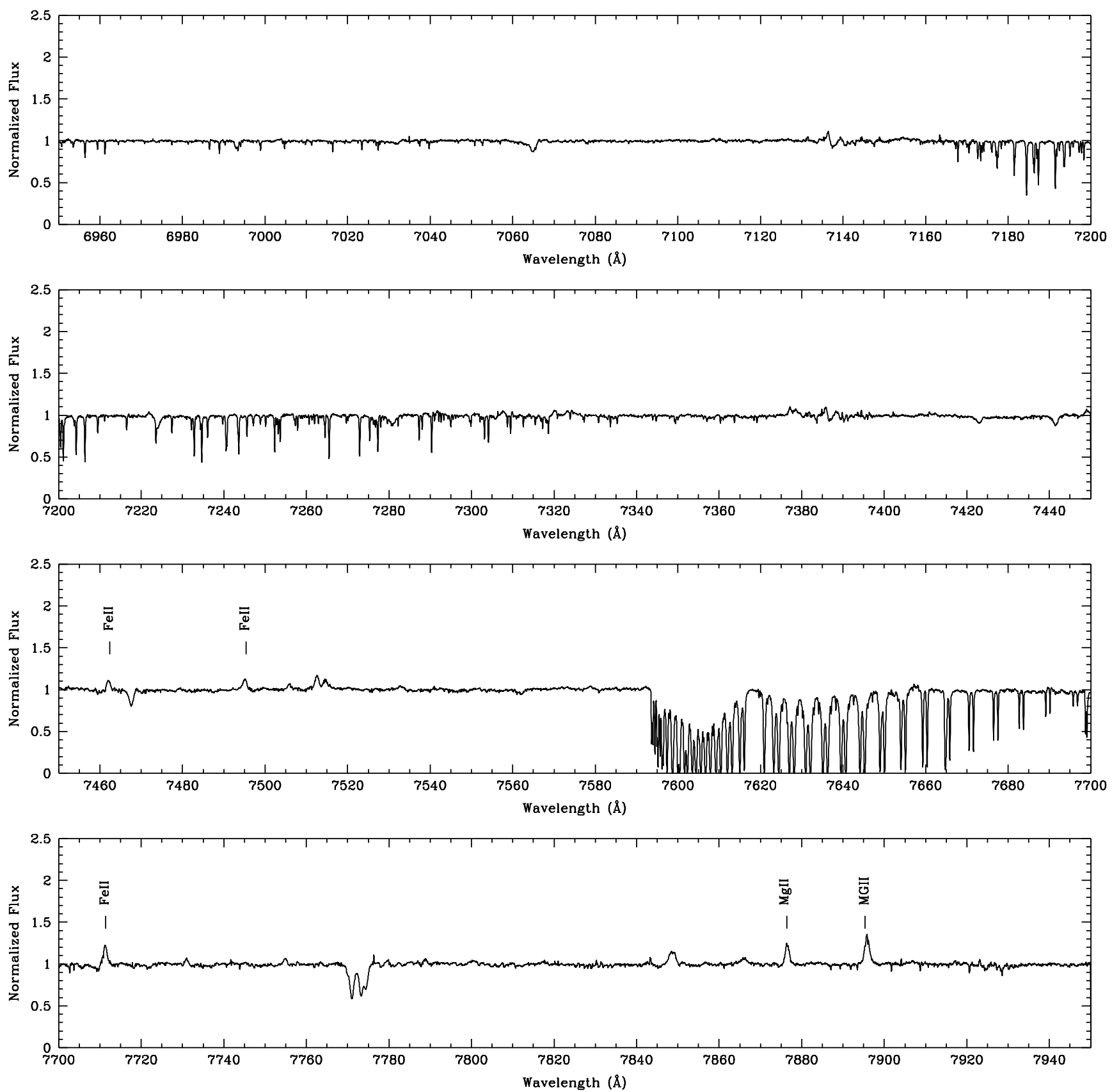

Fig. 4. High resolution spectrum of HR Car obtained with the FEROS spectrograph (continuation).

spherical region close to the star) could be better reproduced than the $\mathrm{H} \alpha$ and $\mathrm{H} \beta$ profiles, since the latter are probably formed in an external region where the spherical assumption is not adequate.

In Fig. 10, we place HR Car in the H-R diagram, showing also the evolutionary tracks of Schaller et al. (1992). Our result indicates that HR Car is located near the $40 M_{\odot}$ track and the line that indicates the total range for the oscillations proposed by van Genderen (2001). The helium abundance that we have found is typical for the He-burning phase. Concerning the temperature, we compared the value derived by us $(10000 \mathrm{~K})$ with those given by Nota et al. (1997), in two different sets of observations near minimum brightness $(20000 \mathrm{~K}$ and $15000 \mathrm{~K})$. These temperature variations are in anti-phase with the light curve: the brighter the star, the lower the temperature (van Genderen 2001). We note also that our estimated value $(10000 \mathrm{~K})$ is not the lowest temperature ever reached by HR Car. From Stromgrem photometry van Genderen (2001) derived the temperature during the light maximum of 1992 to be about $8000 \mathrm{~K}$. From this comparison we conclude that HR Car is crossing the H-R diagram redward on a time scale of years, because of a new SD-phase. 

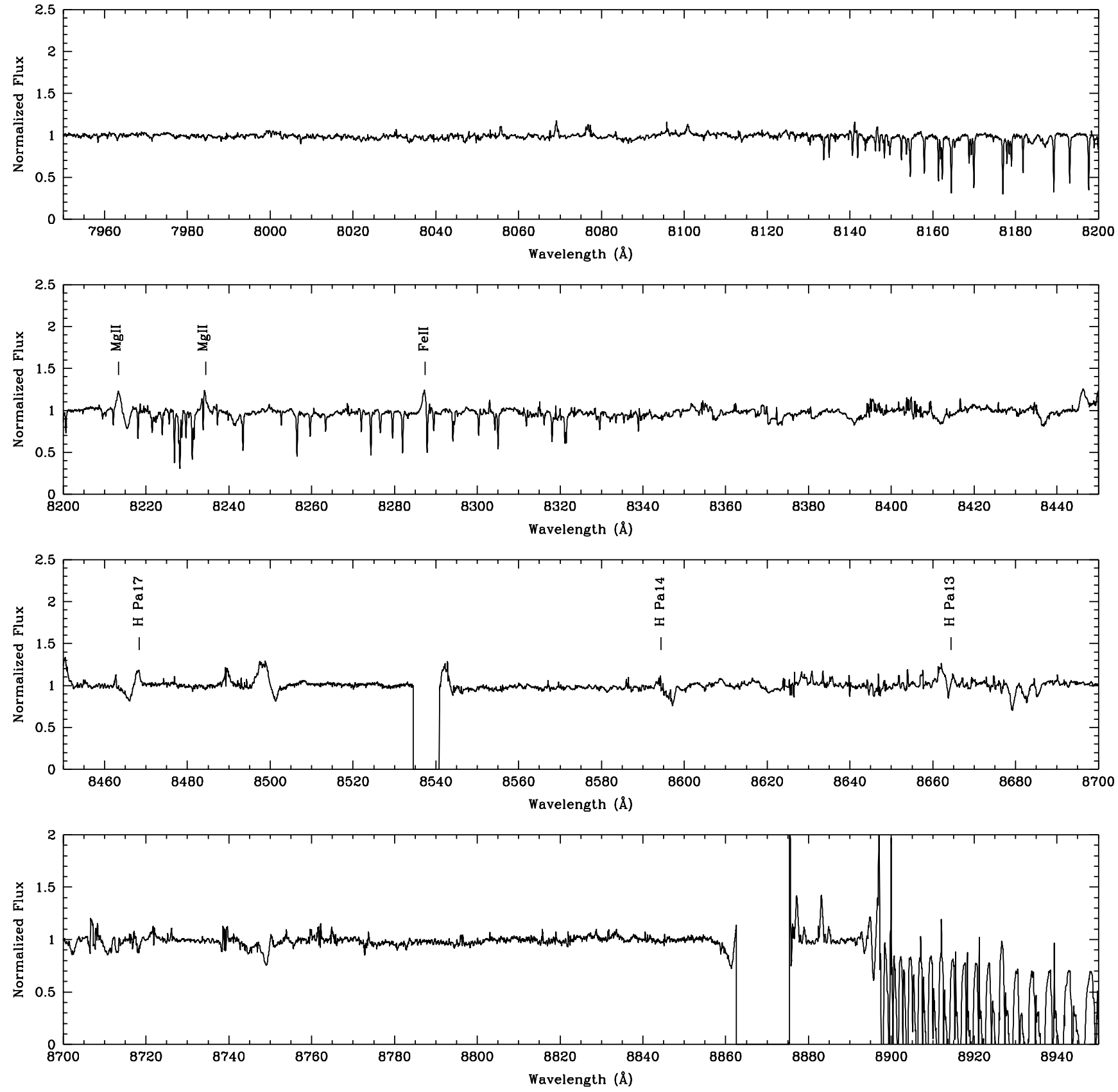

Fig. 5. High resolution spectrum of HR Car obtained with the FEROS spectrograph (continuation).

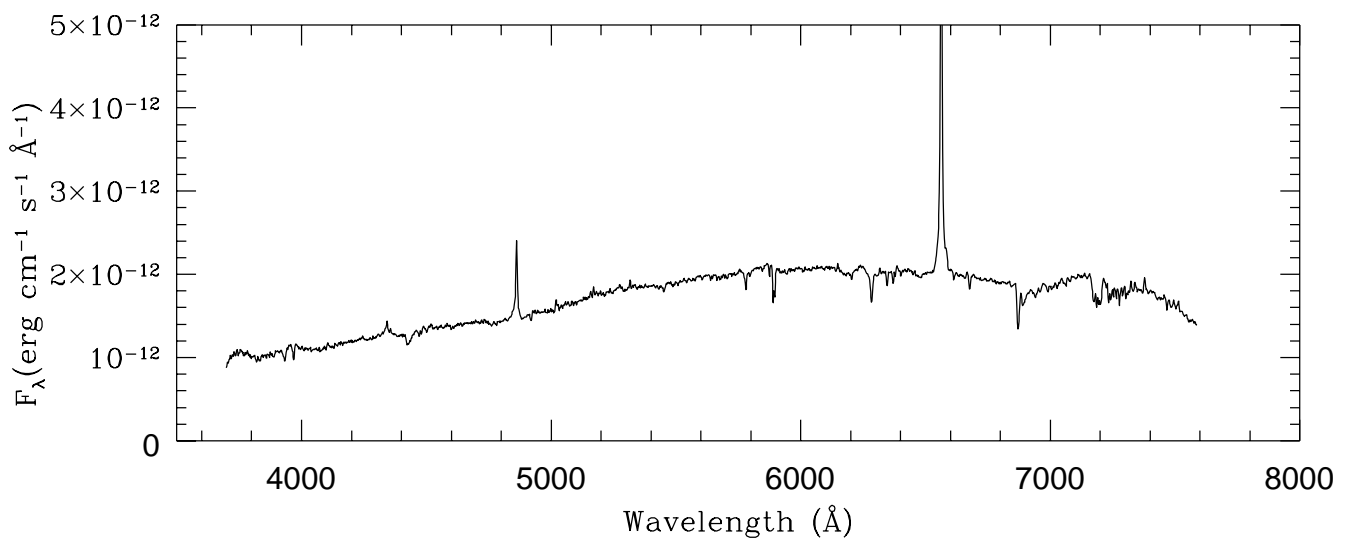

Fig. 6. Low resolution spectrum of HR Car. 

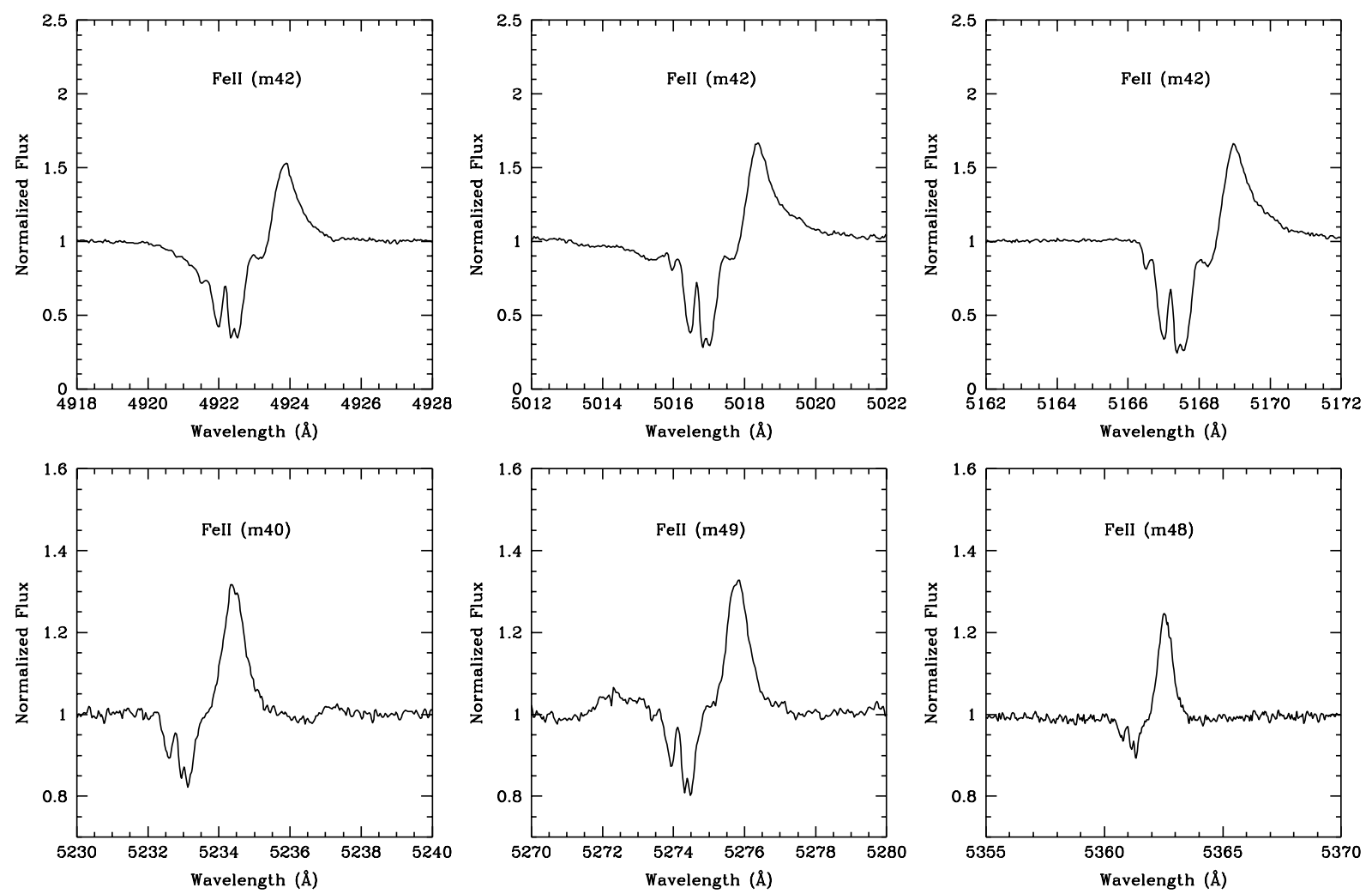

Fig. 7. Some Fe II lines of multiplets $\mathrm{m} 42$ (upper), and m40, m49, and $\mathrm{m} 48$ (lower).
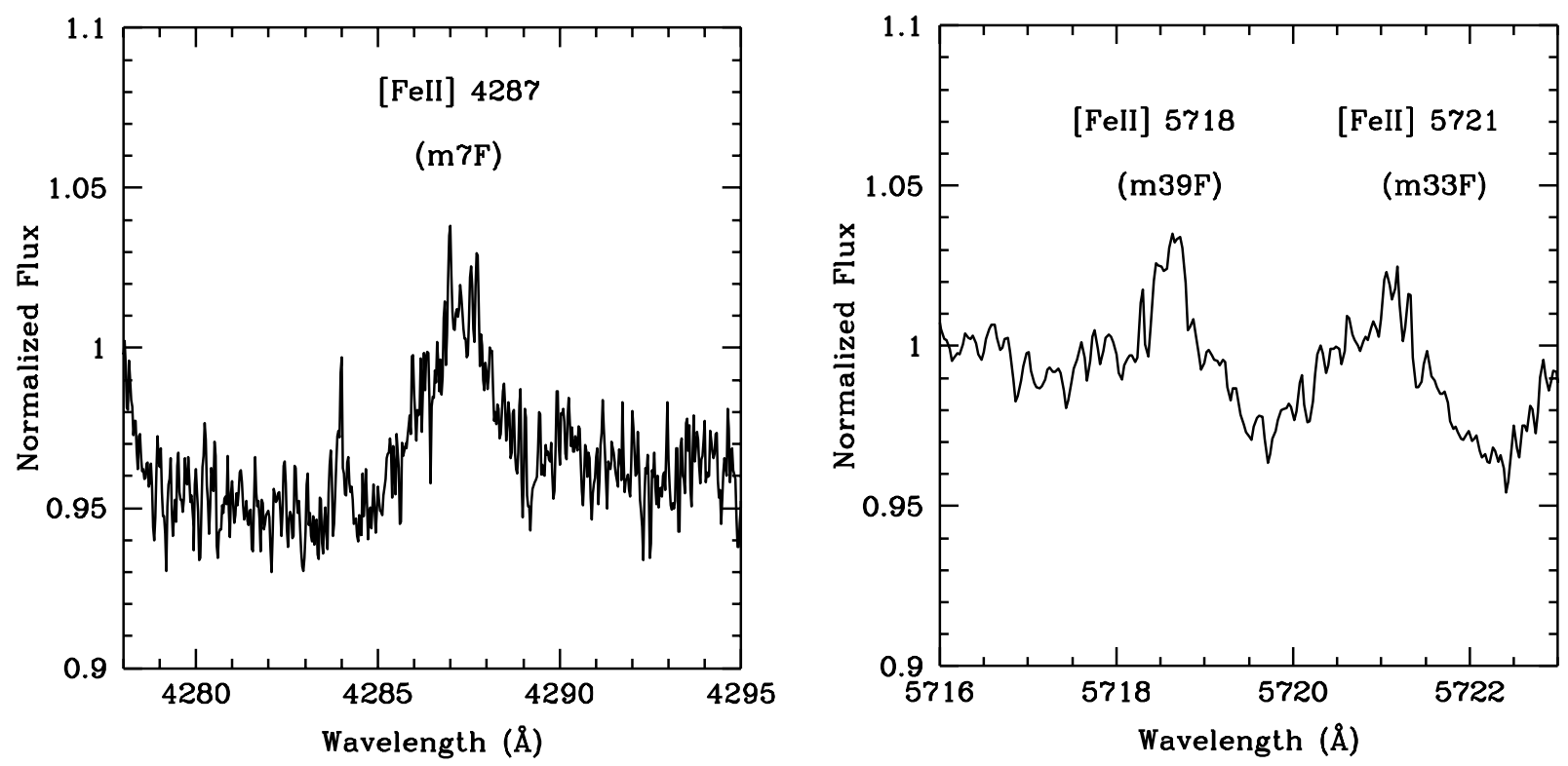

Fig. 8. Observed [FeII] lines.

\section{Conclusions}

We presented recent high resolution spectroscopic data of the LBV HR Car. One of the main characteristics of this object is the Balmer lines showing P-Cygni profiles. In $\mathrm{H} \alpha$ there is a clear structure in the absorption component. These kind of structures were also observed by Hutsemékers et al. (1991) and may be interpreted as an indication of different shells forming around HR Car.
Almost all FeII lines present in the spectra of HR Car also exibit P-Cygni profiles. We clearly see two main components that correspond to radial velocities of $30 \mathrm{~km} \mathrm{~s}^{-1}$ and $100 \mathrm{~km} \mathrm{~s}^{-1}$. Hutsemékers et al. (1991) refer to these two components with pratically the same values of radial velocity.

The direct comparison of our data with the spectra obtained by Nota et al. (1997) in two differents epochs (May 1995 and Jan. 1996), reveals clear spectral variations in 

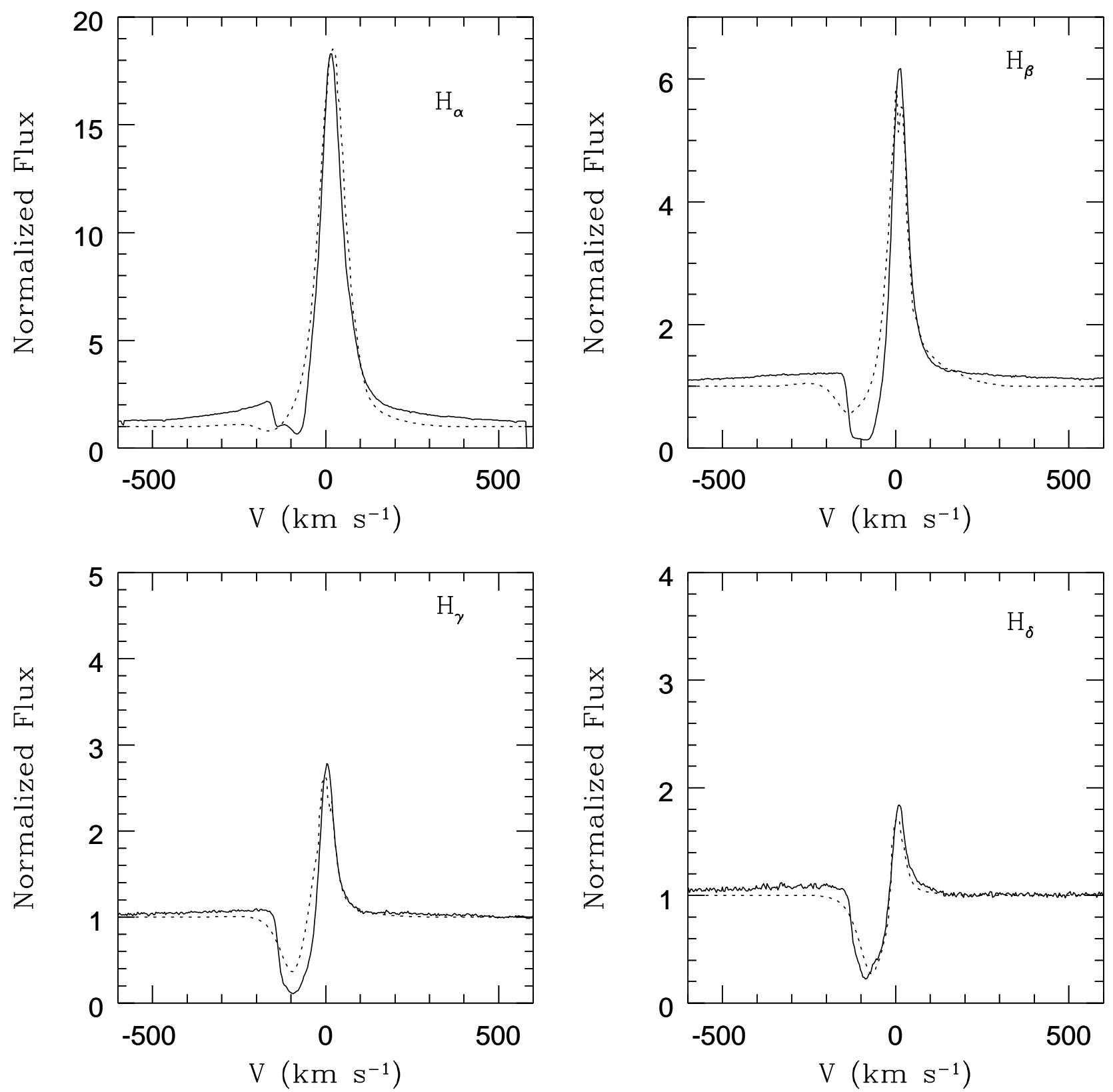

Fig. 9. Observational data (full lines) and best theoretical fittings (dashed lines) for $\mathrm{H} \alpha, \mathrm{H} \beta, \mathrm{H} \gamma$ and $\mathrm{H} \delta$ profiles.

HR Car. The Si II $\lambda \lambda 6347,6371$ lines, which were absent in the first spectra of Nota et al. (1997), arise in their second spectra and are stronger in our spectra. On the other hand, the $\mathrm{N}$ II $\lambda \lambda 6379,6482$ lines decrease in intensity from the first of Nota's data to our spectra (1999). These two phenomena are described by Nota et al. (1997) as an indication of decrease in temperature. Further, they have estimated the temperature using an atmospheric model, and obtained $20000 \mathrm{~K}$ and $15000 \mathrm{~K}$ for the first and second sets of data, respectively.

In the present work we have used a non-LTE model in order to fit the Balmer lines. The intensities and widths of the emission components are well reproduced and the fits can be considered reasonable. The best model corresponds to $\dot{M}=6.5 \times 10^{-5} M_{\odot} \mathrm{yr}^{-1}, T_{\star}=10000 \mathrm{~K}, L_{\star}=5 \times$ $10^{5} L_{\odot}, R_{\star}=350 R_{\odot}$ and $A_{\mathrm{He}}=0.4$. This model locates HR Car near the $40 M_{\odot}$ evolutionary track in the H-R diagram, in a region occupied by the LBVs.

Our temperature values, when compared to those obtained previously by Nota et al. (1997), give strong evidence that HR Car is moving redward across the H-R diagram due to the onset of a new $\mathrm{S}$ Dor phase.

Acknowledgements. It's a pleasure to acknowledge the comments and suggestions of the referee, Dr. A. van Genderen, that improved the first version of this paper. M. Machado thanks FAPERJ (E-26/ 152.573/2000) for financial support. 


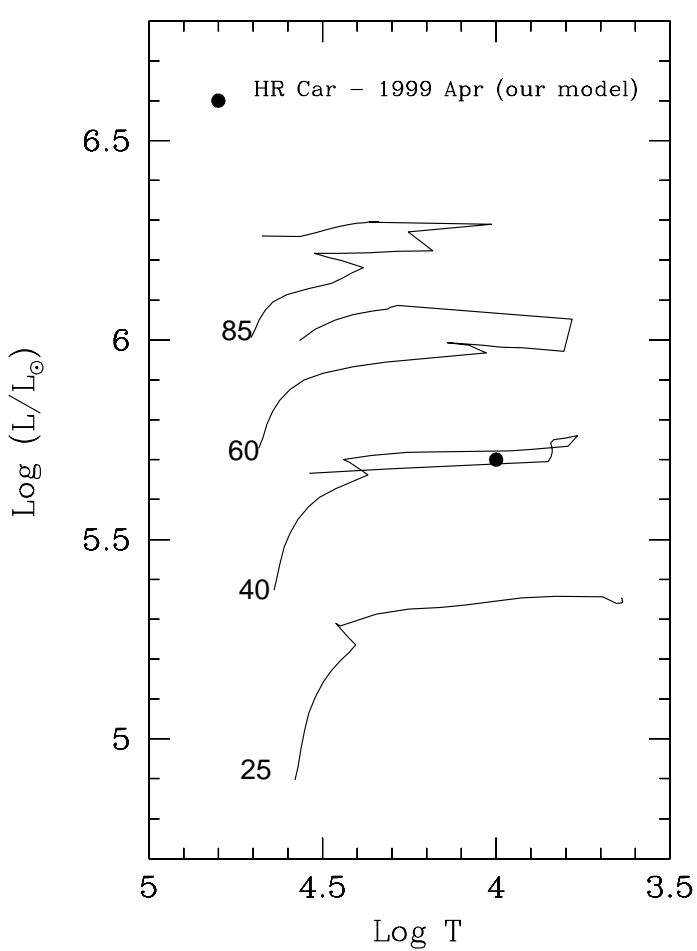

Fig. 10. Theoretical evolutionary tracks of $25 M_{\odot}, 40 M_{\odot}$, $60 M_{\odot}$ and $85 M_{\odot}$ initial main-sequence mass. The full circle locates HR Car according to our result.

\section{References}

Almog, Y., \& Netzer, H. 1989, MNRAS, 238, 51

Auer, L. H., \& Mihalas, D. 1968, ApJ, 151, 311

Berbat, A. P., \& Lambert, D. L. 1978, PASP, 90, 520

Berrington, K. A., \& Kingston, A. E. 1987, J. Phys. B, 20, 6631

Fernandes, M. B., de Araújo, F. X., Pereira, C. B., \& Landaberry, S. J. C. 2001, ApJS, 136, 747

Carlson, E. D., \& Henize, K. G. 1979, Vistas Astron., 23, 213

Clampin, M., Schulte-Ladbeck, R., Nota, A., et al. 1995, AJ, 110,251

de Jager, C., \& Nieuwenhuijzen, H. 1997, MNRAS, 290, 250

de Koter, A., Schmutz, W., \& Lamers, H. J. G. L. M., 1993, A\&A, 277, 561

Drew, J. E. 1985, MNRAS, 217, 867

Garcia-Lario, P., Riera, A., \& Manchado, A. 1998, ApJ, 526, 854

van Genderen, A. M. 2001, A\&A, 366, 508

van Genderen, A. M., Robijn, F. H. A., van Esch, B. P. M., \&

Lamers, H. J. G. L. M. 1991, A\&A, 246, 407 van Genderen, A. M., de Groot, M., \& Sterken, C. 1997, A\&AS, 124,517

Giovanardi, C., Natta, A., \& Palla, F, 1987, A\&AS, 70, 269

Gummersbach, C. A., Zickgraf, F.-J., \& Wolf, B. 1995, A\&A, 302, 409

Hillier, D. J., Crowther, P. A., Najarro, F., \& Fullerton, A. W. 1998, A\&A, 340, 483

Humphreys, R. M., \& Davidson, K. 1994, PASP, 106, 1025

Hutsemékers, D. 1994, A\&A, 281, L81

Hutsemékers, D., \& Van Drom, E. 1991, A\&A, 248, 141

Jefferies, J. T. 1968, in Spectral Line Formation (Blaisdell Publish. Co., Waltham-Massachussets)

Kaufer, A., Stahl, S., Tubbesing, S., et al. 1999, Messenger, 95, 8

Lamers, H. J. G. L. M., Cerruti-Sola, M., \& Perinoto, M. 1987, ApJ, 314, 762

Landaberry, S. J. C., Pereira, C. B., \& de Araújo, F. X. 2001, A\&A, 376, 917

Langer, N., Hamann, W.-R., Lennon, M., Najarro, F., Pauldrach, A. W. A., \& Pulls, J. 1994, A\&A, 290, 819

Lopes, D. F., Damineli Neto, A., \& de Freitas Pacheco, J. A. 1992, A\&A, 261, 482

Machado, M. 1998, in Determinação de parâmetros estelares de objetos massivos: Aplicação as estrelas WCs e LBVs, Ph.D. Thesis

Machado, M., de Araújo, F. X., \& Lorenz-Martins, S. 2001, A\&A, 368, L29

McGregor, P. J., Hyland, A. R., \& Hillier, D. J. 1988, ApJ, 324, 1071

Mihalas, D., \& Stone, M. E. 1968, ApJ, 151, 293

Moore, C. E. 1945, A Multiplet Table of Astrophysical Interest, Part I - Table of Multiplets (revised ed.; Princeton: Princeton Univ. Obs.)

Morris, P. W., Eenens, P. R. J., \& Blum, R. D. 1996, ApJ, 470, 597

Nota, A., Livio, M., Clampin, M., \& Schulte-Ladbeck, R. 1995, ApJ, 448, 788

Nota, A., Smith, L., Pasquali, A., Clampin, M., \& Stroud, M. 1997, ApJ, 486, 338

Schaller, G., Schaerer, D., Meynet, G., \& Maeder, A. 1992, A\&AS, 96,269

Shore, S. N., Brown, D. N., Bopp, B. W., et al. 1990, ApJS, 73,461

Stothers, R. B., \& Chin, C.-w. 1996, ApJ, 468, 842

Szeifert, Th., Humphreys, R. M., Davidson, K., et al. 1996, A\&A, 314, 131

Walborn, N. R., \& Fitzpatrick, E. L. 2000, PASP, 112, 50

Wolf, B. 1989, A\&A, 217, 87

Wolf, B., \& Stahl, O. 1982, A\&A, 112, 111 